Einführung zum Thema

Nephrologe 2020 · 15:4

https://doi.org/10.1007/s11560-019-00396-4

(C) Springer Medizin Verlag GmbH, ein Teil von Springer Nature 2020

\section{Martin Zeier ${ }^{1}$ Joachim Hoyer ${ }^{2}$}

'Sektion Nephrologie, Medizinische Universitätsklinik Heidelberg, Heidelberg, Deutschland

${ }^{2}$ Standort Marburg, Klinik für Innere Medizin, Schwerpunkt Nephrologie, Universitätsklinikum Gießen und Marburg GmbH, Marburg, Deutschland

\title{
Niere und Onkologie
}

Die Onkonephrologie ist ein junges, aber an Beachtung und Bedeutung zunehmendes Feld der Nephrologie mit Schnittstelle zur Onkologie. Ein umfassendes Verständnis der Tumorbiologie und -therapie ist für die die Onkologie begleitende nephrologische Patientenversorgung unerlässlich. Die neue Ära der Immuntherapie hat das Überleben von Patienten mit immunogenen $\mathrm{Tu}$ moren wie dem Nierenzellkarzinom deutlich verbessert, bringt aber auch ein breites Nebenwirkungsspektrum mit sich, welches als autoimmunes Phänomen auch die Niere betreffen kann. Der Themenkreis Onkonephrologie beschäftigt sich darüber hinaus mit epidemiologischen Fragestellungen, wie der Beobachtung gehäuft auftretender Tumorerkrankungen des moderat bis schwerwiegend niereninsuffizienten $\mathrm{Pa}$ tienten, aber auch dem Screening dieser Patienten und der Möglichkeit einer Transplantation im Zustand nach Tumorerkrankung. Letztgenannte Themen werden zunehmend wissenschaftlich systematisch untersucht und die Ergebnisse hoffentlich in Zukunft dazu führen, dass Leitlinien entworfen werden können. Das Gebiet der palliativen Versorgung onkonephrologischer Patienten gewinnt bei zunehmender Anzahl multimorbider onkologischer Patienten an Bedeutung, die Implementierung in den klinischen Alltag ist an vielen Stellen jedoch noch nicht zufriedenstellend umgesetzt. Die Themen dieses Heftes greifen verschiedene Aspekte der Onkonephrologie auf und sollen einen Einblick in dieses spannende Themengebiet ermöglichen.

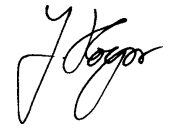

Joachim Hoyer

$M . \xi n$

Martin Zeier

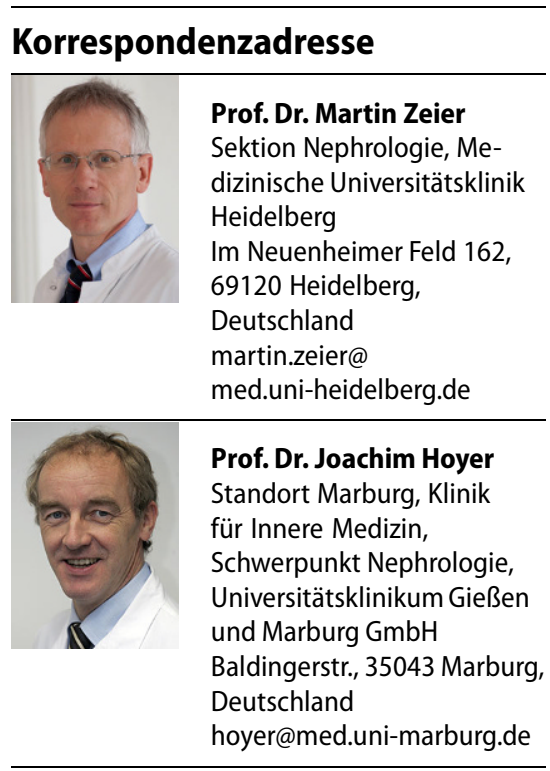

Interessenkonflikt. M. Zeier und J. Hoyer geben an dass kein Interessenkonflikt besteht. 